Editorial

\title{
Acknowledgement to Reviewers of Materials in 2014
}

Materials Editorial Office, MDPI AG, Klybeckstrasse 64, CH-4057 Basel, Switzerland

Published: 7 January 2015

The editors of Materials would like to express their sincere gratitude to the following reviewers for assessing manuscripts in 2014:

Abate, Antonio

Abdallh, A. M. A.

Abdelmalak, Michael Naguib

Abe, Kentaro

Abenojar, J.

Abraham, Wolf-Rainer

Abu-Lebdeh, Yaser

Acet, Mehemet

Aggelis, Dimitrios G.

Agostini, Elisa

Agüero, Alina

Ahn, Yonghyun

Ajersch, Frank

Åkermo, Malin

Aktas, Z.

Al-Abadleh, Hind

Alam, M. Shahria

Albadarin, Ahmad B.

Albin, Sacharia

Alexis, Frank

Allan, Peter

Allinson, David

Almeraya, Facundo

Alonso-Lomillo, M. Asunción

Al-Tabbaa, Abir

Amand, Thierry
Amineh, Reza Khalaj

Ando, Takashi

Andrade, Carmen

Anfodillo, Tommaso

Angelescu, Daniel

Antic, Aleks

António, J.

Anzai, Jun-ichi

Aoki, Hiroyuki

Apostolopoulos, Georgios

Arenillas, Ana

Argyros, Alexander

Arima, Kenta

Arlazarov, Artem

Armentano, I.

Arnold, Donna C.

Aronne, Antonio

Ashraf, Shahid

Ashrafi, Behnam

Asquith, David

Ata, Athar

Avolio, Alberto P.

Azenha, Miguel

Azmi, A. I.

Bai, Jinbo

Baker, Robert
Balázsi, Csaba

Ban, Takayuki

Banerjee, Raj

Bao, Ying

Baragetti, Sergio

Barandiaran, Jose Manuel

Barberá, Joaquín

Barbuta, Costel

Barford, John Patrick

Barhoum, Ahmed

Barkoula, N.-M.

Bartlett, Rebecca

Baskoutas, Sotirios

Bastidas, J. M.

Bastos, Alexandre

Bastos, Ivan Napoleão

Bates, David

Baumeister, Joachim

Baweja, Daksh

Baxavanis, Theocharis

Bednarkiewicz, Artur

Beller, Daniel

Bellucci, Francesco

Belmonte, Thierry

Belviso, Claudia

Ben Khalifa, Nooman 
Benali, Samira

Bencardino, Francesco

Berardi, Valentino Paolo

Berger, Walter

Berglund, Lars

Bermingham, M. J.

Bernardo, Enrico

Bhargava, Suresh Kumar

Bhattacharya, Rahul

Bian, Liming

Bikiaris, Dimitrios

Bikowski, André

Bilotti, Emiliano

Bing, Kong Ling

Birosca, Soran

Boesel, Luciano

Bohner, Marc

Boissiere, Cedric

Bolotov, Leonid

Boningari, Thirupathi Reddy

Boparai, Hardiljeet

Borges, João

Bouwmans, Géraud

Bozhilov, Krassimir N.

Bracke, Lieven

Branco, Fernando

Brandstätter, Christian

Brantley, William A.

Braun, Ulrike

Brennan, Anthony B.

Brischke, Christian

Brockman, Robert A.

Brüning, Karsten

Burkel, Eberhard

Burnley, Stephen

Byun, Joon-Hyung

Cabeza, Aurelio

Cachim, Paulo B.

Calzada, M. Lourdes

Cambier, Francis

Camblor, Miguel A.

Cammidge, Andrew
Campanelli, Sabina Luisa

Campbell, John

Çamurlu, H. Erdem

Cao, G.

Capobianco, John A.

Caporali, Stefano

Carbone, Giuseppe

Cardea, S.

Casalino, Giuseppe

Castanho, Miguel

Castela, A. S.

Castro-Gomes, Joao

Catellani, Marinella

Cavaliere, $\mathrm{P}$.

Cavalli, Andrea

Cavalline, Tara

Cavallini, Mauro

Celano, Umberto

Cesano, Federico

Chadov, Stanislav

Chagnon, Gregory

Chamos, A. N.

Chan, Kwong Yu

Chang, Chih-hung

Chang, Chih-yuan

Chang, Jeong Ho

Chang, S. Y.

Chang-Liao, Kuei-shu

Changyun, Jiang

Chapman, James

Chatel, Gregory

Chatterjee, Sabornie

Chaudhry, Abdul J.

Chen, Changle

Chen, Chih-Ping

Chen, Chun-Hu

Chen, Jem-Kun

Chen, Jian

Chen, Kew-Yu

Chen, Ruei-Tang

Chen, T. M.

Chen, Xiaolong
Chen, Yangyin

Chen, Yue

Chen, Zeng-tao

Chen, Zuliang

Chenal, Jean-Marc

Chiechi, Ryan C.

Chien, Sum Tze

Chigrinov, Vladimir G.

Chipara, Dorina

Chiu, Kong Hwa

Chmielus, Markus

Cho, Man-Gi

Cho, Won-Ju

Choi, Duck-Kyun

Choi, Jae-Hong

Choi, Yoon-Seok

Chowdhury, Sanjib

Christodoulou, C.

Chu, Dewei

Chu, Jian

Chung, Chanmoon

Chung, T. C. M.

Ciardelli, Francesco

Ciardelli, Gianluca

Cinelli, Patrizia

Clarke, Tracey M.

Coakley, Eoin

Cobo, Alfonso

Cochran, Eric W.

Cole, Matt

Coll, Mariona

Collins, Frank G.

Cook, Wayne D.

Copeland, Audrey

Corcione, Carola Esposito

Cordoyiannis, George

Corma, Avelino

Coronas, Joaquín

Corriou, Jean-Pierre

Costa García, Agustín

Courtin-Nomade, Alexandra

Cousins, Brian G. 
Cown, Dave J.

Cran, Marlene J.

Creatore, $\mathrm{M}$.

Criado, $\mathrm{M}$.

Cristiani, Pierangela

Cullen, Jonathan M.

Czerwinski, Frank

Dai, Jian Guo

Dalgarno, Kenneth

Damborenea, Juan

Daniel, Laurent

Dapino, Marcelo J.

Darling, Seth

Das, Diganta B.

Das, Suman

Datsyuk, Vitaliy

Daus, Birgit

Davidovits, Joseph

Davidson, Jane H.

Davies, Peter

Davim, Paulo

De Belie, Nele

De Brito, Jorge

De Geest, Bart

De Rooij, Mario

De Sio, Luciano

De Tommasi, Domenico

Defouw, John

Delale, Feridun

Deleporte, Emmanuelle

Delgado, Rafael

Deng, Hongbing

Deng, Shiqiang

Devaraj, Amutha

Dewan, M. Washim

Dhere, Neelkanth G.

Diaz, Urbano

Dickinson, Alex

Didangelos, Athanasios

Diéguez, Oswaldo

Dietrich, D.

Dimiev, Ayrat M.
Dimitrijevic, Nada M.

Dinia, Aziz

Distaso, Monica

Djurdjevic, Mile B.

Dobrzański, L. A.

Domenek, Sandra

Domenici, Valentina

Dong, F.

Dong, Hongbiao

Dong, Liang

Dorozhkin, Sergey V.

Douki, Thierry

Dourado, Fernando O. Queirós

Dragic, Peter

Dragoni, Eugenio

Driver, Julian

Duarte, Ana Rita C.

Dubey, Ashish

Dufossé, Laurent

Dujardin, Christophe

Dunn, J. B.

Duong, Hai M.

Dupin, Jean Charles

Eamon, Christopher

Easton, Mark

Ebara, Ryuichiro

Ebel, Thomas

Ebendorff-Heidepriem, Heike

Ebong, Abasifreke

Edalati, Kaveh

Efthymiou, Evangelos

Eick, Sigrun

Eizenberg, Moshe

Eklund, Per

Elaissari, Abdelhamid

Elbaz, Lior

El-Kaderi, Hani M.

Elliman, Robert

Elliott, Simon

Elsentriecy, Hassan

Endres, Frank

Enfedaque, Alejandro
Engelberg, Dirk

Engler, Olaf

Eramo, Giacomo

Erathodiyil, Nandanan

Erdem, Emre

Espallargas, García S. J.

Espino, Daniel M.

Esposito, Emanuela

Evangelista, Luís

Evlyukhin, Andrey B.

Fabio Zuluaga, Hector

Fabregat, Azael

Fabrizio, Quadrini

Falletta, Ermelinda

Fedel, Mariangela

Feliu, Sebastian

Felser, Claudia

Feng, Xian

Fernandes, Emanuel M.

Fernandes, Susete

Fernandez, A. I.

Fernsler, Jonathan

Ferrari, Maurizio

Ferreira, José Maria da Fonte

Ferrier, E.

Ferro, Gabriel

Février, Sébastien

Fierro, J. L. G.

Filonovich, Sergej

Fletcher, Ashleigh

Focacci, Francesco

Fogelström, Linda

Follain, Nadège

Foot, Peter

Francesconi, Grazia M.

Fraternali, Fernando

Fratini, Livan

Fray, Derek J.

Friák, Martin

Fridkin, Vladimir

Frignani, Alessandro

Fujita, Kyoko 
Fujita, Toyohisa

Fujiwara, Kohei

Fukada, Toshiyuki

Fukui, Kunihiro

Funahashi, Ryoji

Fürbeth, Wolfram

Furlani, Edward P.

Furue, Hirokazu

Gabbitas, Brian

Gaddam, Raghuveer

Galán-Mascarós, José Ramón

Gallego, Sergi

Galstian, Tigran

Ganguly, Supriyo

Gao, Junqi

García, Hermenegildo

García, Juan

Garcia-Escorial, A.

García-Etxarri, Aitzol

García-Gutiérrez, Mari-Cruz

Garratt, Elias

Garrett, David J.

Garrett, Simon J.

Geddis, Demetris L.

Gerber-Lemaire, Sandrine

Gernay, Thomas

Gerôme, F.

Gerós, Hernâni

Gervasini, Antonella

Gherardi Hein, Paulo Ricardo

Gheribi, Aimen E.

Ghiotti, A.

Ghosh, Gargi

Giannakos, Konstantinos

Gil, Francisco Javier

Giovambattista, Nicolas

Girousi, Stella

Gkotsis, Petros

Glaeser, J. A.

Godinho, Maria

Goel, Saurav

Gomez-Romero, Pedro
González, María de las Nieves

González, S.

González-Fonteboa, Belén

Gonzalez-Marcos, M. Pilar

Goodall, Russell

Goodhew, Steve

Gordon, Wong

Grajcar, A.

Granberg, Hjalmar

Grandini, Carlos R.

Granozio, Fabio Miletto

Grasso, Salvatore

Graule, Thomas

Gray, Kimberly A.

Greco, Antonio

Greenly, Justin M.

Gregg, J. M.

Gregorczyk, Keith

Grimaldi, C.

Grimsdale, Andrew Clive

Grujicic, M.

Guan, Dongsheng

Gubner, Rolf

Gueunier-Farret, M.

Guldiken, Rasim

Guldner, Norbert W.

Guo, Youguang

Guo, Zhanhu

Gupta, Nikhil

Gupta, Rishi

György, Enikö

Ha, Kiryong

Habert, Guillaume

Habijan, Tim

Haick, Hossam

Haider, Waseem

Hallberg, Håkan

Halle, Thorsten

Hamilton, Jeremy

Hamzaoui, Rabah

Han, Daewoo

Han, Min-Cheol
Han, Yousoo

Han, Zhao Jun

Hanaor, Dorian

Hanawa, Takao

Hane, Kazuhiro

Hangai, Yoshihiko

Hanna, Junichi

Hara, Masayuki

Harding, D. R. K.

Hargreaves, Justin

Harkin, Damien

Hassan, Marwa

Hassel, Achim Walter

Hattori, Haroldo T.

Havenith, Remco

Hayakawa, Tohru

Hayashi, Yoshihiko

Hayes, Daniel J.

$\mathrm{He}$, Bo

Healy, Noel

Heath, Andrew

Heilmann, Andreas

Hellier, Alan Keith

Henniges, Ute

Herting, Gunilla

Hezel, Dominik

Hickey, Stephen G.

Hidalgo, J. Ignacio

Highland, Matthew J.

Hilliou, Loic

Hinderberger, Dariush

Hinds, G.

Hintze, Wolfgang

Hirama, Hirotada

Hiziroglu, Salim

Ho, Johnny Chung Yin

Ho, Wen-Fu

Holmström, Stefan

Hong, Cheng-Shong

Hong, Yi

Hossain, Khandaker M. A.

Hosur, Mahesh V. 
Howard, Ian A.

Howarter, John

Hsiao, Vincent

Hsu, Hsiu-Fu

$\mathrm{Hu}$, Jinlian

$\mathrm{Hu}$, Jonathan

$\mathrm{Hu}$, Ning

$\mathrm{Hu}$, Yuh-Chung

Huang, Baoshan

Huang, Huang-Chiao

Huang, Jie

Huang, Mao-Jung

Huang, Wei Min

Huang, Yuanding

Huang, Yu-Tzu

Hubbe, Martin A.

Hubert, Madlen

Hubner, Jens

Hudhomme, Piétrick

Hug, Eric

Hughes, Taylor L.

Humbeeck, Jan Van

Hun Ryu, Sung

Hüttner, Sven

Hyun, Hoon

Ibana, Don

Ibris, Neluta

Iezzi, Giovanna

Ijima, Hiroyuki

Ingarao, Guiseppe

Ingham, Jason

Iniesta, Jesús

Inoue, Katsutoshi

Ionov, Leonid

Islam, Syed Kamrul

Ito, Seigo

Ivanov, Dimitri A.

Ivanov, Ilia N.

Iwasaki, Tomohiro

Jabbari, Mohammad Ali

Jabbarzadeh, Ahmad

Jackson, Nathan
Jakli, Antal

Jakubowicz, Ignacy

Jalali, Said

Janshoff, Andreas

Janus, Magdalena

Jayakumar, Rangasamy

Jayasinghe, Suwan

Jeng, Chyuan-hwan

Jeong, Doo Seok

Jeong, Han Mo

Jeong, Moon Seok

Jeong, Yongchae

Jestin, Jacques

Jezierska, Julia

Jiang, Jiang

Jiang, Liudi

Jin, Kejia

Jo, Young Min

Johnson, Scooter D.

Jones, Lathe

Jordon, Brian J.

Jose, Joby Kochumalayil

József, Karger-Kocsis

Jung, Sungmoon

Kadota, Kazunori

Kaewunruen, S.

Kako, Tetsuya

Kalaskar, Deepak

Kamegawa, Takashi

Kan, Daisuke

Kaneco, Satoshi

Kang, Chulhun

Kang, Lifeng

Kang, Thomas

Kannan, Bobby

Karalekas, Dimitris

Karg, Matthias

Kartsonakis, I. A.

Kasper, Kurtis

Katmis, Ferhat

Katsamenis, Orestis L.

Katta, Lakshmi
Kaufman, Laura J.

Kaufman, Michael

Kawanago, Takamasa

Kawashima, Shiho

Ke, Changhong

Kelley, Michael J.

Kepczynski, Mariusz

Kergoat, Loïg

Kessler, Horst

Kevern, John

Khaliq, Wasim

Kharlampieva, Eugenia

Khatib, Jamal

Khun, Nay Win

Kidoaki, Satoru

Kiefer, Björn

Kilian, Kristopher A.

Kim, Chang-Soo

Kim, Dae Eun

Kim, Do Hyun

Kim, Dong-Pyo

Kim, Doseok

Kim, Eunkyoung

Kim, Gap-Yong

Kim, Hak Yong

Kim, Hee Chan

Kim, Heesun

Kim, Hyeong Joon

Kim, Jae-Hoon

Kim, Jin Kuk

Kim, Jung Ho

Kim, Kwang S.

Kim, Seung-Joo

Kim, Taehwan

Kim, Yoong Ahm

Kimura, A.

Kimura, Munehiro

Kimura, Yoshiharu

Kindt, James T.

King, Michael R.

Kishi, Yoichi

Kivisäkk, Ulf 
Klemperer, Walter G.

Klenk, Andreas

Klepka, Andrzej

Klose, Christian

Knight, Jonathan

Kobelke, Jens

Köferstein, Roberto

Kofinas, Peter

Kojima, Chie

Kolasinski, Kurt W.

Kolppo, Kari

Komnitsas, Kostas

Konegger, Thomas

Kontturi, Eero

Koodali, Ranjit

Koos, Antal A.

Korai, Hideaki

Koricho, Ermias Gebrekidan

Korposh, Serhiy

Korshunov, M. M.

Korstanje, Ties J.

Koseki, Toshihiko

Koskinen, Jari

Kotadia, Hiren

Kotobuki, Masashi

Koukouzas, Nikolaos

Kourkoumelis, Nikolaos

Koutsoukos, Petros

Kowalska, Ewa

Kraehmer, Andrea

Kranzmann, A.

Kriezis, Emmanouil E.

Krishnan, Sadagopan

Krupski, Aleksander

Kuder, Katherine G.

Kuehn, Christian

Kühn, Fritz E.

Kukli, Kaupo

Kuktaite, Ramune

Kulichikhin, Valery

Kunioka, Masao

Kuo, Chao-Yin
Kuo, Dong-Hau

Kuo, Shiao-Wei

Kuo, W. T.

Kuo, Y. K.

Kwitkowski, Lech

Kwok, C. T.

Kwon, Il-Keun

Kymissis, Ioannis

Kyzas, George Z.

Labergere, Carl

Labrincha, J. A.

Lai, Chao

Lai, Chao-Sung

Lalevée, Jacques

Lambert, Paul

Lamberti, Gaetano

Lammens, Nicolas

Lampke, Thomas

Lan, Wen-How

Langhals, Nicholas B.

Lannutti, John J.

Lantada, Andres Diaz

Lanzoni, Luca

Larobina, Domenico

Laschat, Sabine

Lattuada, Marco

Lavrentovich, Oleg D.

Law, David

Lazzara, Giuseppe

Le Gac, Pierre-Yves

Lebaron, Richard

Leblanc, Roger

Leclerc, Corey

Leclerc, Nicolas

Lee, Bongmook

Lee, Cheng-Kang

Lee, Cheol Jin

Lee, Dongwon

Lee, Jae-chun

Lee, Jehyun

Lee, Juliana Tsz Yan

Lee, Mi-Jung
Lee, Sang Bok

Lee, Seung Hee

Lee, W.

Lee, Woojin

Lee, Ya-Ju

Lehmann, Jean

Lehmhus, Dirk

Leinenbach, Christian

Lekakh, Simon

Leon, Marta

Letizia, Rosa

Leung, Pui Tak

Levänen, Erkki

Lévêque, Patrick

Lewis, Gladius

$\mathrm{Li}$, Bin

Li, Dingsheng

Li, Gang

Li, Guo Liang

Li, Guoqiang

Li, Kang

Li, Luhua

Li, Qiliang

Li, Quan

Li, Robert K. Y.

Li, Weiyang

Li, Xiang

Li, Yang

Lianos, Panagiotis

Lichtensteiger, Céline

Liew, S. L.

Lin, Chhiu-Tsu

Lin, Dong

Lin, Hong-Ping

Lin, I.

Lin, Jiang-Jen

Lin, Jing-Jenn

Lin, Jui-Teng

Lin, Song

Lin, Wei-Ting

Lin, Yuqing

Ling, Tung-Chai 
Lionetto, Francesca

Lira-Cantu, Monica

Liu, Bo-Tau

Liu, Chuan-Hsi

Liu, Fwu-Hsing

Liu, Hongfei

Liu, Hongwei

Liu, Jingfei

Liu, Jinxuan

Liu, Rui

Liu, Yongliang

Lo Presti, Davide

Loke, Vincent

Lokuge, Weena

Lombillo, Ignacio

Lonjon, Antoine

López, Enrique Vera

Lord, Megan S.

Lorenzo, Donati

Lorenzo, E.

Lorite, I.

Lousteau, Joris

Lu, Cheng

LU, Li

Lu, Weigang

Luches, Armando

Ludwig, Ralf

Lugo, M.

Luo, Yanhua

Luyckx, Geert

Luzio, Alessandro

Lvov, Yuri

Ma, Dexin

Ma, John Z.

Maassen, Jesse

Machida, Masashi

Maclachlan, Mark J.

Maeda, Tetsuro

Mafi, Arash

Magliulo, Maria

Mahajan, Sujit

Mahesh, B. V.
Maheshwari, Vivek

Maierhofer, Christiane

Major, Matthew J.

Mallavia, R.

Malucelli, Giulio

Mamba, Bhekie B.

Manciu, Felicia

Manfredi, Diego

Mangialardi, Teresa

Mani, Gopinath

Mann, Amanda K. P.

Margarido, Fernanda

Margulis, Walter

Markos, Christos

Markou, Giorgos

Marsal, Lluis F.

Martinelli, Enzo

Martínez, Adriana

Martins, Rodrigo

Martí-Vargas, J. R.

Marton, Zsolt

Masai, Hirokazu

Masami, Ikeyama

Mascher, Peter

Materer, Nicholas F.

Mathaudhu, Suveen N.

Mazzolani, Federico

McCloskey, Bryan D.

McDonald, Armando G.

Mecartney, Martha

Mehl, Georg

Meier, Urs

Meneghetti, Giovanni

Menendez, J. L.

Meng, Yi

Mercolini, Laura

Mertens, Anne

Meulenberg, Robert W.

Mhaede, Mansour

Michailidis, Nikolaos

Mihashi, Hirozo

Milanese, Daniel
Minteer, Shelley D.

Miomandre, Fabien

Mistakidis, Euripides S.

Mittal, Vikas

Mizutani, Fumio

Młyniec, A.

Mobed-Miremadi, Maryam

Mock, Adam

Möhwald, Helmuth

Mokaya, Robert

Molchan, I. S.

Molina, Pablo

Møller, Uffe

Monge, M. A.

Monteiro, Regina C. C.

Montemor, Maria Fátima

Monticelli, Cecilia

Moreira, M. Teresa

Moreno Tost, Ramón

Moriana, Rosana

Morkoc, Hadis

Morris, Michael

Mortensen, Andreas

Motz, Günter

Mou, Chung-Yuan

Mougenot, J.

Müller, Bert

Muller, Robert N.

Muller-Buschbaum, Peter

Munoz-Pinto, Dany

Murcia-López, S.

Musso, Simone

Mutin, Hubert

Naher, Sumsun

Nair, Lakshmi S.

Nair, Sriramya D.

Naji, Majd Al

Najm, Husam

Nakagaito, Norio

Nakayama, Masaharu

Nam, N. D.

Nam, S. 
Nara, Yoshitaka

Naraghi, M.

Natali, Maria Elia

Naya, Shin-ichi

Neimark, Alexander V.

Neri, Giovanni

Nespoli, Adelaide

Ngai, T.

Nganga, Sara

Nien, H. H.

Nohut, Serkan

Nomura, Kotohiro

Novakovic, Jelica

Nunes, Sandra

O'Connor, É.

Obalová, L.

Obidziński, Sławomir

Odijk, Theo

Ogihara, Hitoshi

Ogino, Hiraku

Ogle, Kevin

Oh, Byung Hwan

Ohara, Keishi

Ohno, Hajime

Ohshima, Tatsuya

Okawa, Seigo

Okimoto, Yoichi

Oliva, Leone

Olmi, Giorgio

Onishi, Hiroshi

Onwudili, J. A.

Ordóñez, Salvador

Oskouei, Reza

Osório, Wislei R.

Osten, H. Jörg

$\mathrm{Ou}$, Jianzhen

Oudah, Fadi

Pace, Andrea

Pacheco-Torgal, Fernando

Pan, Tongyan

Pan, Xuan

Pan, Zhongqi
Panagiotaras, Dionisios

Panesar, Daman

Pantazopoulos, George

Papadopoulou, K.

Papavassiliou, Dimitrios V.

Park, Soo-Jin

Park, Yeong-Joon

Parker, Sandra

Parsons, Jason

Parviz, Babak Amir

Paschalis, Eleftherios I.

Pasek, Matthew

Pasupathy, Praveen

Patenaude, Mathew

Patolsky, Fernando

Patten, John

Patzke, Greta Ricarda

Pavlidis, Ioannis

Peacock, Anna

Pedersen, Henrik

Pedone, Alfonso

Pehlken, Alexandra

Peisert, Heiko

Pellicer, Eva

Pelton, Robert H.

Peng, Bo

Pereira de Oliveira, L. A.

Perez, M.

Pérez, Marco Antonio

Peroukidis, Stavros D.

Perrot, Arnaud

Pervak, Volodymyr

Peters, Kara

Peterson, Karl

Petrlova, Jitka

Petruzzelli, Domenico

Peukert, Wolfgang

Pey, Kin Leong

Peyvandi, Amirpasha

Philipose, U.

Phillion, Andre

Photinos, Demetri
Pickett, Matthew D.

Pietrzyk, Bozena

Pilat, Francesco

Pingarron, Jose Maria

Pirker, Stefan

Piro, Benoit

Pita, Marcos

Plank, Johann

Plechkova, Natalia

Pokhrel, Suman

Pomianowski, Michal

Poncelet, Denis

Poon, S. J.

Popat, Ketul C.

Popov, Anatoliy V.

Populoh, Sascha

Portehault, David

Potgieter, Herman

Power, Ian M.

Prade, Rolf

Pradhan, Sangram K.

Prashanth, Konda G.

Pretsch, Thorsten

Prosandeev, Sergey

Provis, John L.

Pucci, Andrea

Puche, Regino Sáez

Puertas, Francisca

Puga, H.

Pujo, Mariacinta C.

Pullammanappallil, Pratap C.

Puppi, Dario

Qiao, Kun

Qiu, Liang

Quiquempois, Yves

Radhika, Madhav A.

Ragab, Kh. A.

Rahbar, Nima

Rahier, Hubert

Ramaker, David E.

Ramanujam, Prabhu

Ramesh, Aramandla 
Ramirez, Anibal

Ramirez, Carmen

Rangan, Vijaya

Ras, Robin H. A.

Rasekh, Manoochehr

Ratna, Banahalli R.

Ravelli, Davide

Rawls, Henry

Ray, Asim

Rebelo, Susana L. H.

Reclaru, Lucien

Ree, Moonhor

Refait, Ph.

Reijnders, Lucas

Reinhardt, Hans-Wolf

Reis, P. N. B.

Reiss, Guenter

Ren, Yuhang

Requena, Guillermo Carlos

Rhamdhani, M. Akbar

Ribeiro, Maria S.

Ribot, François

Riccardi, Leonardo

Richards, Bryce S.

Richardson, Hugh $\mathrm{H}$.

Riggs, Brian C.

Ritala, Mikko

Ritcey, Anna

Rob Sharif, Muhammad Ali

Robertson, Peter K.

Robinson, Sara C.

Rodrigues, Danieli

Rodríguez, M. A.

Rodríguez-Valverde, M. A.

Roeb, Martin

Roggendorf, Hans

Rolon-Garrido, Victor

Romanato, Filippo

Rometsch, Paul

Ronca, S.

Ronkainen, Niina J.

Ros, Blanca M.
Rossi, Claire

Rost, Marcel J.

Rouabhia, Mahmoud

Rowland, Doug

Rowson, Neil

Roy, Anish

Ruhl, Aki Sebastian

Russell, Richard

Ruys, Andrew

Ryan, James G.

Sa'ncheza, Luis

Sabo, Ronald

Sadeghi, Alireza

Saleh, Haitham

Salvador, James

Sanchez, Florence

Sánchez-Ferrer, Antoni

Sánchez-Jiménez, Pedro E.

Sandri, Giuseppina

Santana, Juan J.

Santos, Catarina

Sardella, Eloisa

Sato, Soshi

Sawpan, Moyeenuddin A.

Sazio, Pier

Schaefer, Dirk J.

Schlangen, Erik

Schledjewski, R.

Schmidt, Daniel F.

Schneider, Raphael

Schneider, Raphaël

Schultz, B. F.

Schulze, Ulrich

Schuman, Thomas

Schwarzkopf, J.

Scotognella, Francesco

Scott, Jason

Sedev, Rossen

Segawa, Hiroyo

Seguí Palmer, Concepción

Seifalian, Alexander

Seifert, Gerhard
Seisenbaeva, Gulaim A.

Semba, Takeshi

Sen, Tushar

Seppälä, Jukka

Sepúlveda-Escribano, Antonio

Sesana, Raffaella

Shafaei, Shahram

Shah, Ramille

Sharif-Khodaei, Zahra

Shehata, Medhat

Shen, Li

Sherratt, Michael J.

Shi, Xianming

Shian, Samuel

Shibasaki-Kitakawa, Naomi

Shih, Po-Jen

Shimosawa, Tatsuo

Shin, Hyung Shik

Shinagawa, Tsutomu

Shing Ching, Tak

Shiraishi, Yasuhiro

Shiue, Sham-Tsong

Shokuhfar, Tolou

Shon, In-Jin

Shtein, Max

Shubhakar, Kalya

Sidiras, Dimitris

Siegel, Jakub

Sierra, Teresa

Silberschmidt, Vadim

Sillanpaa, Mika

Silva, H. M. Ribeiro Dias da

Silva, Rui A.

Silveyra, Josefina

Simar, Aude

Simka, Wojciech

Simões, Sónia

Singh, Mahi

Singh, Ranjan

Sinha, Arijit

Sista, Prakash

Skirtach, André G. 
Slowing, Igor I

Sluckin, Tim J.

Slussarenko, Sergei

Smått, Jan-Henrik

Smay, Jim E.

Smith, Mark

Sobolkina, Anastasia

Södergård, Anders

Söderholm, Patrik

Soin, Navneet

Soldano, Caterina

Sommer, Michael

Song, Fei

Song, Yi

Sorgente, Donato

Soroushian, Parviz

Sorrentino, Andrea

Southam, Gordon

Spadea, Saverio

Spiesz, Przemek

Spigarelli, Stefano

Spychaj, Tadeusz

Srinivasa, Arun

Srivastava, Alok M.

Stannarius, Ralf

Steinbach, Ingo

Stepuk, Oleksandr

Stevenson, Adam J.

Stokes, Kevin L.

Stolichnov, Igor

Strangwood, Martin

Straumal, Boris

Straumal, Boris B.

Streli, Christina

Strömme, Maria

Strzhemechny, Yuri

Stuhr, Uwe

Stumpe, Joachim

$\mathrm{Su}$, Lihong

Suárez, Isaac

Suarez-Garcia, Fabian

Sue, Hung-Jue
Sugden, Kate

Sukhishvili, Svetlana

Sumner, Joy

Sun, Henghu

Sun, Hongqi

Sun, Kien Wen

Sun, Shi-Peng

Sun, Tao

Sun, Xiao

Sunol, Joan Josep

Suryanarayana, C.

Suryanto, Benny

Sutor, Alexander

Suzuki, Daisuke

Swain, Michael

Swaminathan, Viswanathan

Światowska, Jolanta

Syrrokostas, G.

Sytschev, Alexander E.

Tabrizian, Roozbeh

Taglietti, Angelo

Tailhades, Philippe

Takamura, Yayoi

Takeuchi, Masato

Tamai, Toshiyuki

Tanaka, Keiji

Tang, Luping

Tang, Ning

Taratula, Oleh

Tariq, Mohammad

Tauer, Johannes

Taylor, Doris

Teixeira, Fernando

Tena-Zaera, Ramon

Teng, Hsisheng

Teoh, Lay Gaik

Terentjev, Eugene M.

Theivasanthi, T.

Thomas, C.

Thomas, Carlos

Thomas, Stuart

Thöming, Jorg
Thompson, Van P.

Thomson, Andrew

Tian, Yanqing

Tigli, Onur

Tisserat, Brent

Tiwari, Shruti

Tjong, S. C.

Tkacz-Smiech, Katarzyna

Todaro, Simona

Tofail, Syed A. M.

Tokoro, Chiharu

Tomashchuk, Iryna

Tomich, John

Tomlinson, Douglas

Torimoto, Tsukasa

Torrenti, Jean Michel

Toupance, Thierry

Traisnel, Michel

Tricot, Gregory

Trimmel, Gregor

Tripp, Carl P.

Troles, Johann

Tsao, Chung-Chen

Tsao, L. C.

Tsay, Chien-Yie

Tsay, L. W.

Tse, Ming-Leung Vincent

Tsivilis, $\mathrm{S}$.

Tsouknidas, A.

Tsubota, Toshiki

Tsujimoto, Takashi

Tuch, Bernard E.

Tukker, Arnold

Tulliani, Jean-Marc

Tulunoglu, Ibrahim

Tung, Tran Thanh

Tung, Vincent C.

Uher, Ctirad

Uji-I, Hiroshi

Umeton, Cesare

Upadhyay, Piyush

Uraoka, Yukiharu 
Uygun, Basak E.

Vaiano, Vincenzo

Vairo, Giuseppe

Valente, Isabel

Valentini, Luca

Van den Eeckhout, Koen

Van Gemert, Dionys

Van Riessen, Arie

Van Stam, Jan

Van Tittelboom, Kim

Van Velzen, Ulphard Thoden

Vannier, Rose-Noëlle

Vardaxoglou, Yiannis

Várnai, Anikó

Vázquez, Enric

Vedrine, Jacques

Venkataraman, Shrinivas

Verge, Pierre

Vicente, J. de

Vigier, Karine

Víllora, Gloria

Virtanen, Sannakaisa

Visai, Livia

Vizcaíno, Arturo J.

Vladimirov, Ivaylo

Vodenitcharova, Tania

Vogel, Michael

Vogtländer, Joost

Voisey, K. T.

Von der Mark, Klaus

Vonau, W.

Vorndran, Elke

Vos, Paul de

Vozmediano, María

Vygranenko, Y.

Wagner, Travis

Waite, Matthew M.

Wallinder, Inger Odnevall

Wan, Kai Tai

Wan, Kai-Tak

Wang, Ce

Wang, Chien-Lung
Wang, Chong-Min

Wang, Li

Wang, Min

Wang, Ran

Wang, Shanfeng

Wang, Shaobin

Wang, Xiao-Qian

Wang, Xungai

Wang, Zhonglin

Ward, L. P.

Ward, Liam

Watts, Benjamin

Wei, Suying

Weimer, Paul J.

Weinstein, Dana

Weiss, Jason

Wennerber, Ann

Whang, Wha-tzong

White, Timothy

Wille, Kay

Williams, Vance E.

Wilson, Mark. R.

Wiskott, Anselm H. W.

Witt-Enderby, Paula A.

Wondraczek, Karin

Wong, Danny K. Y.

Wu, C. H.

$\mathrm{Wu}$, Jiamin

Wu, Kevin

Wu, Mao-Sung

$\mathrm{Wu}$, Shin-Tson

$\mathrm{Wu}, \mathrm{X} . \mathrm{F}$.

$\mathrm{Wu}$, Xijia

Wurmehl, S.

Xia, Wei

Xue, Wei

Yamabe-Mitarai, Yoko

Yamamoto, Akio

Yamamoto, Go

Yamauchi, Satoshi

Yamauchi, Yusuke

Yan, Ning
Yan, Xiaoli

Yang, H. Y.

Yang, Jinglei

Yang, Kap Seung

Yang, Renbo

Yang, Yong

Yannick, Mugnier

Yasuzawa, Mikito

Yazyev, Oleg

Yeh, Jui-Ming

Yeh, Min-long

Yentekakis, I. V.

Yeow, Edwin K. L.

Yi, Jiabao

Ying, Shang-Ping

Yokoi, Toshiyuki

Yokoyama, Kenji

Yoo, Dong Jin

Yoon, Seong-Ho

Yoon, Ung Chan

Yoshinari, Masao

Yoshino, Toshihiko

Yoshitake, Hideaki

Yousif, Belal F.

Yu, Chang-Feng

$\mathrm{Yu}$, Dingshan

Yu, Long

$\mathrm{Yu}$, Yanghai

Yun, Sang-Won

Zakutayev, Andriy

Zaleski, Jeffrey

Zanetti, Mariachiara

Zaraska, Leszek

Zehetner, Christian

Zeinert, Andreas

Zeugolis, Dimitrios

Zhang, Lifeng

Zhang, Mingxing

Zhang, $\mathrm{Xu}$

Zhang, Yan

Zhang, Zuhua

Zheng, Wei 
Zhou, Hongyu

Zhou, Jinming

Zhou, Jun

Zhu, Weihong

Zhu, Xiaoshan
Zhu, Youfeng

$\mathrm{Zhu}, \mathrm{Yu}$

Zhuravleva, Ksenia

Zimmermann, Uwe

Zitoune, Redouane
Zomorodian, A.

Zou, Ying

Zubarev, Eugene

Zuin, Stefano

Zych, Eugeniusz

(C) 2015 by the authors; licensee MDPI, Basel, Switzerland. This article is an open access article distributed under the terms and conditions of the Creative Commons Attribution license (http://creativecommons.org/licenses/by/4.0/). 